\title{
TEST YOURSELF ANSWERS
}

These are possible answers to the quizzes at the end of chapters 4 and 5 . They are not definitive: there are often multiple ways to get the required result.

\section{CHAPTER 4}

1. To find subdivided properties, we want any letter after the number, which we can find either by adding the character class $[\mathrm{A}-\mathrm{Za}-\mathrm{z}]$ : to our previous grep:

$$
\text { grep -E "^[0-9]+[A-Za-z]" Balls-Pond-road.txt }
$$

This returns one property, 134A, where a cabinet maker is listed. We can also make grep case insensitive with the $-i$ flag:

$$
\text { grep -Ei "^[0-9]+[a-z]" Balls-Pond-road.txt }
$$

This returns the same result with a bit less typing.

2. Because using the *.txt syntax aggregates all the results from the text files, the distinction between files will be lost. You can operate on all files in a directory one by one by creating a 'loop'. That is beyond the scope of this book, but a web search for 'bash loop' will show the syntax for this.

$$
\text { grep -Ev "^[0-9]"*.txt | less }
$$

3. To filter out the cross streets, you can pipe one grep $-v$ to another. This is a quick way to test some regular expressions. However, it is 
much easier to write the results of this command to a new file called, say, unnumbered-addresses.txt:

$$
\text { grep -Ev "^[0-9]" *.txt > unnumbered-addresses.txt }
$$

You can then clean the file up with a mixture of regex and manual deletions.

\section{CHAPTER 5}

1. grep -E "\bMrs \b" all-b-streets.xml |grep -Eo $",[\wedge]+,<" \mid$ sort | uniq $-\mathrm{c}$ | sort $-\mathrm{nr}$ | less grep -E "\bMiss $\backslash \mathrm{b} "$ all-b-streets.xml |grep -Eo $",[\wedge]+,<" \mid$ sort | uniq $-\mathrm{c} \mid$ sort $-\mathrm{nr}$ | less

This was our solution but it can probably be improved upon.

2. By definition, this is your choice, but here is a single address from Bacon Street which we have marked up more fully:

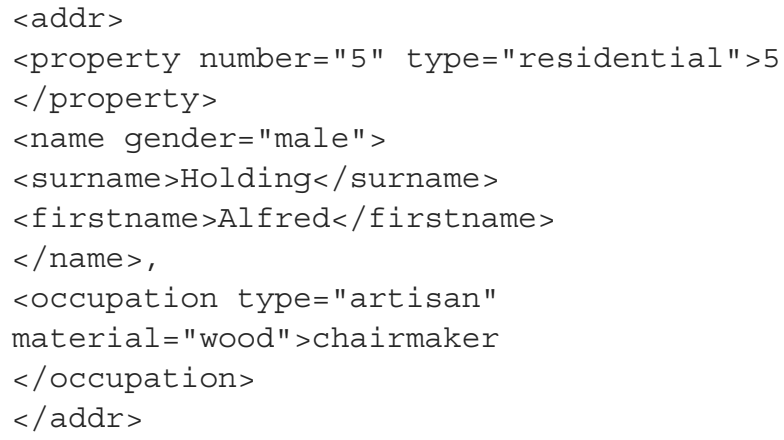

3. With markup added, you can grep for the opening tag, followed by any text, followed by the opening tag. For example, this will list all of the occupations:

grep -EO "<Occupation.+</occupation>" filename.xml 